\title{
Impact of environmental factors on alloimmunity and transplant fate
}

\author{
Leonardo V. Riella, ${ }^{1}$ Jessamyn Bagley, ${ }^{2}$ John lacomini, ${ }^{2}$ and Maria-Luisa Alegre ${ }^{3}$ \\ 'Schuster Family Transplantation Research Center, Renal Division, Brigham and Women's Hospital, Boston, Massachusetts, USA. ${ }^{2}$ Department of Developmental, Molecular and Chemical Biology, Tufts University \\ School of Medicine, Sackler School of Biomedical Sciences Programs in Immunology and Genetics, Boston, Massachusetts, USA. ${ }^{3}$ Department of Medicine, University of Chicago, Chicago, Illinois, USA.
}

\begin{abstract}
Although gene-environment interactions have been investigated for many years to understand people's susceptibility to autoimmune diseases or cancer, a role for environmental factors in modulating alloimmune responses and transplant outcomes is only now beginning to emerge. New data suggest that diet, hyperlipidemia, pollutants, commensal microbes, and pathogenic infections can all affect T cell activation, differentiation, and the kinetics of graft rejection. These observations reveal opportunities for novel therapeutic interventions to improve graft outcomes as well as for noninvasive biomarker discovery to predict or diagnose graft deterioration before it becomes irreversible. In this Review, we will focus on the impact of these environmental factors on immune function and, when known, on alloimmune function, as well as on transplant fate.
\end{abstract}

The strength and potency of an alloimmune response following organ transplantation are determined by the frequency and avidity of $\mathrm{T}$ cells for alloantigens expressed by the graft, a process that depends largely on the number of genetic disparities between the donor and the recipient. The possibility that environmental factors may further modulate the strength of the antitransplant immune response has recently gained interest. Although the impact of environmental factors may not be readily identifiable in heavily immunosuppressed patients, it may become more relevant in patients on minimal immunosuppression, or in the few patients in whom immunosuppression can be withdrawn. In this Review, we will focus on environmental factors that can impact the kinetics of graft rejection in animal models, namely high-salt diet (HSD); high-fat diet (HFD) and associated hyperlipidemia; pollutants; and microbes.

\section{HSD and transplant fate}

While diet has long been considered an important risk factor for pathologies such as heart disease, the demonstration of its importance in immunity is recent. Among dietary components, salt $(\mathrm{NaCl})$ has drawn attention based on its potential effects on both innate and adaptive immunity. Although salt is an essential element of the human diet, the daily amount of salt consumed exceeds the recommended intake and has been associated with greater risk of hypertension and stroke (1). Below, we will discuss the effects of salt on innate and adaptive immunity.

Salt, monocytes, and the lymphatic system. $\mathrm{NaCl}$ is the main determinant of the extracellular fluid volume of the body and is tightly regulated by pressure receptors, the sympathetic nervous system, and hormones, including the renin-angiotensin-aldosterone system; however, recent studies have demonstrated that isotonicity is not stable in tissues. Lymphoid tissues were found

Conflict of interest: The authors have declared that no conflict of interest exists. Reference information: / Clin Invest. 2017;127(7):2482-2491.

https://doi.org/10.1172/JCI90596. to be hyperosmolar (2), and ${ }^{23} \mathrm{Na} \mathrm{MRI}$ demonstrated greater sodium accumulation in the skin of patients with refractory hypertension (3). Macrophages appear to play a role in buffering excess salt intake, preventing expansion of the extracellular volume and consequently blunting surges in blood pressure. Titze and colleagues showed that rats fed an HSD accumulated hypertonic sodium in the interstitial space of the skin, leading to hyperplasia of the lymphatics via activation of tonicity-responsive enhancer binding protein (TonEBP) in interstitial mononuclear phagocyte system (MPS) cells and production of angiogenic VEGF-C (4). Deletion of TonEBP in MPS cells prevented this compensatory response upon HSD exposure and led to higher blood pressure in mice (5). Similarly, blocking VEGF-C receptor impaired the expansion of skin lymphatics and induced salt-sensitive hypertension (5). In another study, higher sodium content in the skin enhanced the immune response against the protozoan parasite Leishmania major through stimulation of nuclear factor of activated T cells 5 (NFAT5) in macrophages, promoting activation of classical macrophages that exhibited effector function via NOS2 (6). Lastly, high salt impaired M2 macrophage differentiation through inhibition of mTOR/AKT signaling, resulting in impaired wound healing in mice (7). In sum, increased salt promotes inflammatory macrophages while inhibiting antiinflammatory macrophages (Figure 1). Whether sodium retention in skin further lowers the threshold for immune activation and promotes rejection in vascularized composite tissue transplants that contain skin remains to be determined.

Salt and adaptive immunity. Increased salt intake may induce pathogenic T cells and impair immune regulation. In vitro, $\mathrm{NaCl}$ $(40 \mathrm{mM})$ promoted Th17 induction in both murine and human $\mathrm{T}$ cells through a mechanism dependent on p38 MAPK, NFAT5, and serum glucocorticoid kinase 1 (SGK1) activation (8). Further, HSD accelerated the onset and severity of murine experimental autoimmune encephalomyelitis (EAE) $(8,9)$. Mechanistically, high salt induced SGK1, which promoted IL-23R expression and enhanced Th17 cell differentiation in vitro and in vivo. In mice with SGK1deficient T cells, EAE severity was abrogated with impaired Th17 


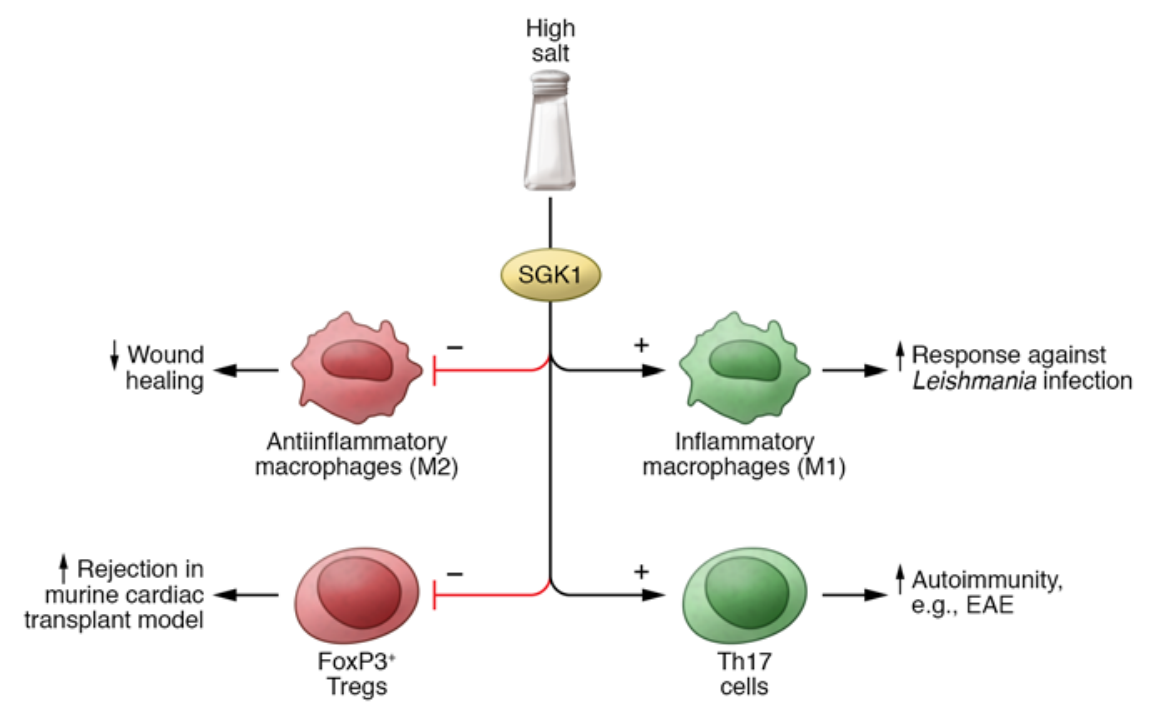

Figure 1. Effects of high salt on innate and adaptive immunity. High-salt diet may stimulate macrophages to differentiate into classical macrophages (M1), potentiating their effector function through NFAT5 activation and increasing the immune response against Leishmania infection. In a murine model of wound healing, high salt inhibits M2 differentiation through inhibition of mTOR/AKT signaling, leading to delayed wound healing. In adaptive immunity, high salt may potentiate Th17 cell differentiation and exacerbate disease in the experimental autoimmune encephalomyelitis (EAE) model. In a murine model of cardiac transplantation, highsalt diet is deleterious to immune regulation and precipitates rejection by reducing the proportion of Tregs via SGK1 activation and consequent Fox01/Fox03a phosphorylation. cell differentiation despite HSD (9). Because the gut is the primary site exposed to HSD, one hypothesis is that HSD may modify intestinal microbiota, leading to autoimmunity. The microbiota does seem to play a contributory role in EAE initiation (10), but the exact effect of HSD in the microbiota is unknown. In humans, an observational study that estimated sodium intake based on sodium urine excretion in two independent cohorts of patients with multiple sclerosis (MS) showed that higher sodium intake was associated with increased MS exacerbation in a multivariate model adjusted for other parameters (11). Whether altering salt intake may reduce MS exacerbation remains to be determined.

High-salt exposure also increases alloimmunity (Figure 1). In a single-mismatch MHC class II cardiac transplant model, Safa et al. demonstrated that HSD accelerated allograft rejection despite a lack of changes in serum sodium concentration or mean blood pressure (12). The rejection in the HSD group was associated with reduced percentage and proliferation of $\mathrm{CD}^{+}{ }^{+} \mathrm{FoxP}^{+}$Tregs, leading to a lower Treg/T effector ratio than in control animals. Allografts exhibited significantly lower Treg infiltration in the HSD group compared with the control group, but no difference in Th17 cells. Nonetheless, T cell SGK1 mediated the deleterious effect of HSD on Tregs by triggering phosphorylation of FoxO1/ FoxO3a, thereby sequestering these important Treg transcriptional factors in the cytoplasm (12). A similar deleterious effect of salt on Tregs was observed in a xenogeneic graft-versus-host disease (x-GVHD) model and in an adoptive transfer model of experimental colitis (13). Hernandez et al. demonstrated that high sodium induced Treg IFN- $\gamma$ production via SGK1, impairing Treg suppressive function (13). IFN- $\gamma$ neutralization restored Treg inhibitory function. Thus, salt affects both Treg stability and function, and an HSD impairs immune regulation, accelerating rejection in a murine cardiac transplant and an $\mathrm{x}-\mathrm{GVHD}$ model. Whether manipulating dietary salt intake alters rejection rates in humans remains to be determined.

\section{HFD, hyperlipidemia, and alloimmunity}

Atherosclerotic coronary artery disease causes end-stage heart disease in approximately $40 \%$ of all patients requiring a heart trans- plant (14). Atherosclerosis is now considered to be a chronic inflammatory disease (15-18), and humans with atherosclerosis exhibit increased serum levels of inflammatory cytokines. Hyperlipidemia also develops in $50 \%$ of heart transplant patients after the first year after transplantation and in as high as $95 \%$ of patients within 5 years (19). The standard treatment for hyperlipidemia is administration of cholesterol-lowering statins (20). In spite of treatment, twothirds of transplant patients remained dyslipidemic, and a significant number of heart transplant patients are statin intolerant (21, 22), leaving the majority of transplant patients with significantly elevated lipid levels. Insofar as hyperlipidemia promotes inflammation, the influence of this condition on recipient antidonor responses could have a major impact on transplant outcomes.

Evidence that hyperlipidemia affects transplant outcome can be found in chronic rejection. Chronic rejection of heart transplants results in cardiac allograft vasculopathy (CAV). A history of ischemic heart disease resulting from hyperlipidemia-associated coronary artery disease is a major recipient characteristic associated with cardiac allograft rejection after the first year after transplantation (14). Increases in the ratio of triglycerides to HDL were correlated with increased frequency and severity of CAV, and preexisting dyslipidemia at the time of heart transplantation was found to be a significant risk factor for the development of CAV (23). Progression of CAV correlated with increases in total cholesterol and LDL (24), and total cholesterol levels after 1 year after transplantation may have prognostic value in determining which patients will develop CAV (25). Consistent with the idea that hyperlipidemia negatively affects transplant outcome, reducing lipids can reduce the incidence of rejection. Statins decreased the incidence of acute rejection, improved 1-year survival, and reduced the development of CAV (26). However, in some studies, other cholesterol-lowering drugs did not improve transplant outcome (27). While improved transplant survival may be related to the ability of statins to lower lipid levels, statins also have immunomodulatory properties that are independent of lipid-lowering effects (28-30) in monocytes (31-39) and T cells (33, 34, 40, 41). Indeed, when treated with lipid-lowering agents, mice with normal lipid levels exhibited prolonged transplant survival (26). 


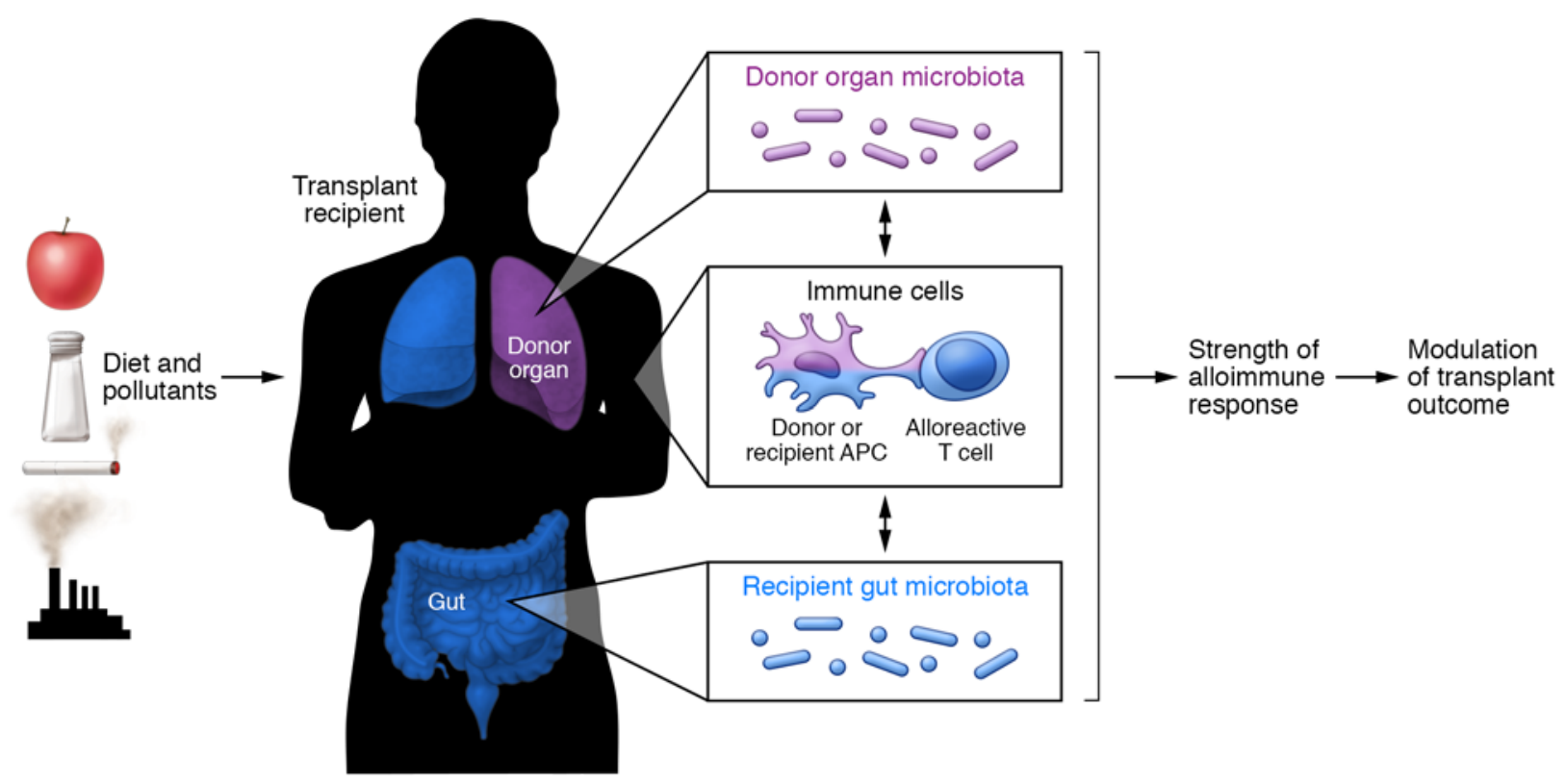

Figure 2. Model for the modulation of transplant outcomes by the microbiota as a central and rapid integrator of host-environmental pressures. Select microbial communities can enhance the ability of APCs to activate alloreactive T cells and accelerate graft rejection. Diet and pollutants can modify the composition of colonizing microbial communities, which may in part relay their immunomodulating effects. Moreover, the reciprocal crosstalk between the immune system and the microbiota implies that the alloimmune response and the immunosuppressive drugs taken by transplant hosts may trigger additional feedback loops between microbial changes and the strength of the alloimmune response, eventually impacting graft outcome.

Modeling effects of hyperlipidemia on transplant survival. Several murine models of altered plasma lipids have been developed for the study of atherosclerosis, including apolipoprotein E-deficient (ApoE-deficient) mice (42). Feeding ApoE-deficient mice an HFD increased serum cholesterol (42-45), although these mice did not become obese or hyperglycemic, making it possible to study the effects of high plasma lipid levels without other confounding comorbidities that have independent effects on immunity $(46,47)$.

ApoE-deficient mice have systemic immune system changes, similar to those seen in hyperlipidemic patients including increased Th1 and Th17 responses $(17,18,48)$ and changes in immune responses to pathogens (49-51). Using ApoE-deficient mice as a model of recipient hyperlipidemia revealed profound effects on transplant rejection and its regulation (52). Hyperlipidemic recipients exhibited accelerated rejection of fully mismatched cardiac allografts and acutely rejected MHC class II-mismatched transplants that typically undergo chronic rejection only. Acute rejection in hyperlipidemic hosts resulted from alloreactivity, as syngeneic grafts survived long-term. These changes in the kinetics of rejection were not due to the reported immunomodulatory effects of ApoE (45), as hyperlipidemic ApoE-sufficient mice fed an HFD also acutely rejected MHC class II-mismatched transplants. Moreover, the degree of hyperlipidemia positively correlated with an increase in rejection kinetics. Thus, hyperlipidemia leads to accelerated rejection independently of obesity and hyperglycemia. Accelerated rejection of minor antigen-mismatched cardiac grafts was also observed in mice on an HFD compared with mice on a nutrient-matched low-fat diet (53). Together, these data point to a role for hyperlipidemia in transplant rejection.

Mechanisms of transplant rejection in hyperlipidemia. Th1 cells are typically considered the main $\mathrm{CD}^{+} \mathrm{T}$ cell subset that mediates rejection. In hyperlipidemic mice, however, Yuan et al. observed an increased frequency of IL-17-producing T cells in the periphery. A significant number of IL-17-producing graft-infiltrating cells were observed in MHC class II-mismatched as well as fully mismatched cardiac allografts transplanted into hyperlipidemic recipients (45). In contrast, IL-17-producing graft-infiltrating cells were not observed in hearts undergoing rejection in control recipients. Survival of allogeneic hearts in hyperlipidemic recipients was significantly prolonged when IL-17 was neutralized or genetically deleted. These data suggest that the condition of hyperlipidemia promotes a rejection response that involves a Th17 component not observed in mice with normal lipid levels.

Cholesterol in $\mathrm{T}$ cell activation. $\mathrm{T}$ cell activation and clonal expansion require significant synthesis of cholesterol-containing membranes (54). T cells accumulate cholesterol either through cellular synthesis or through the uptake of exogenous cholesterol via LDL receptor (LDLR) or alternate receptors. Cellular cholesterol levels are regulated by transcription factors that promote cholesterol efflux (the liver X receptors, $\operatorname{LXR} \alpha$ and LXR $\beta$ ) and degrade LDLR or through factors that promote cholesterol accumulation (SREBPs). Perturbation of cellular cholesterol homeostasis markedly alters $\mathrm{T}$ cell responses. $\mathrm{T}$ cell-specific deletion of the ATP-binding cassette transporter G1 (ABCG1), which promotes cellular cholesterol efflux, enhanced T cell receptor (TCR) signaling (55). SREBP-deficient $\mathrm{CD}^{+} \mathrm{T}$ cells displayed defective proliferation and deficient clonal expansion during viral infection (56). Inhibition of ACAT1, a cholesterol esterification enzyme, led to increased $\mathrm{CD}^{+} \mathrm{T}$ cell cytotoxicity, TCR clustering, and TCR signaling (57). Further, cholesterol binds to the TCR- $\beta$ chain (58), and the ratio of cholesterol to inhibitory cholesterol derivatives can potentiate TCR-mediated activation (59). 
Cholesterol also plays a role in $\mathrm{T}$ cell polarization following activation. While oxysterol-induced LXR prevented Th17 generation by inhibiting aryl hydrocarbon receptor-dependent (AHR-dependent) IL-17 transcription (60), non-LXR agonist oxysterols induced ROR $\gamma \mathrm{t}$, the master transcriptional element controlling Th17 development (61). Adding oxysterols to naive T cells under polarizing conditions promoted the development of Th17 cells (61). Treg functions seem particularly sensitive to cholesterol levels. Inhibition of 3-hydroxy-3-methyl-glutaryl-coenzyme A reductase (HMGCR), which controls cholesterol synthesis, prevented Treg proliferation and impaired Treg function (62), and Abcg1 deletion increased Treg proliferation (63). In the LDLR knockout model of hyperlipidemia, increases in the splenic frequency of Tregs were correlated with changes in phenotype and their functional inability to control atherosclerotic lesions (64).

Effects of cholesterol on antigen-presenting cells. Cholesterol also plays an important role in membrane maintenance that is critical for proper antigen-presenting cell (APC) function $(65,66)$. Abcg1 $1^{-1}$ macrophages, which have impaired cholesterol efflux and elevated cholesterol levels, exhibited an intrinsic bias toward M1 polarization with increased NF- $\kappa$ B activation (67) and enhanced proinflammatory cytokine production $(68,69)$. Oxidized LDL (ox-LDL) induced autophagosome formation, MHC-II expression, and phosphorylation of SYK in macrophages (70). Oxidized cholesterol promoted the differentiation of monocytic precursors into phenotypically mature dendritic cells (DCs) (71), and treatment of DCs in vitro with ox-LDL was associated with upregulation of the costimulatory molecules CD86 and MHC-II and downregulation of the coinhibitory molecule programmed death ligand-1 (PD-L1) (72). While these data suggest a role for cholesterol in APC function, results have been contradictory for antigen presentation. DC subsets isolated from hyperlipidemic ApoE-deficient mice did not have altered antigen-presenting function $(73,74)$. It has been suggested that ApoE-deficient macrophages are more activated $(75,76)$ and have reduced scavenging capacity when compared with control cells (77). In HFD-induced obesity models, increased percentages of CD11 $c^{\text {hi }}$ DCs and $\mathrm{CD} 11 \mathrm{c}^{\mathrm{lo}} \mathrm{MHC}-\mathrm{II}^{+} \mathrm{B} 22 \mathrm{O}^{+}$plasmacytoid DCs were found in the spleen; however, these cells had decreased stimulatory capacity (78). DCs isolated from C57BL/6 mice fed an HFD for 8 weeks showed no changes in their ability to stimulate $\mathrm{CD} 4{ }^{+} \mathrm{CD} 25^{-}$effector cells in vitro (79). Thus, overall antigen presentation in hyperlipidemic mice remains unchanged or slightly impaired. These results differ from those of a recent study in which APCs were found to have greater stimulatory capacity if animals were fed an HFD for over 12 weeks, when obesity-induced inflammation is prominent (53). Also, in this study, control mice were fed a nutrient-matched low-fat diet, rather than normal chow, possibly revealing a lower stimulatory capacity of control APCs. Additionally, different diets alter the microbiota differently, and, as described below, different microbial communities have different capacities to tune APCs. How APCs integrate tuning by the microbiota with direct consequences from hyperlipidemia remains to be investigated.

Hyperlipidemia and Tregs. Bagley et al. found that hyperlipidemia profoundly alters Tregs. $\mathrm{CD}^{+}{ }^{+} \mathrm{FoxP}^{+}$Tregs were significantly expanded in hyperlipidemic mice; however, these cells downregulated expression of CD25 (52), a finding that resolves the contradictions in previous studies in ApoE-deficient mice $(64,80,81)$, where authors have reported either increased or decreased frequencies of Tregs depending on whether FoxP ${ }^{+} \mathrm{CD} 25^{-}$cells were measured. Bagley et al. also observed that Tregs derived from hyperlipidemic animals were less suppressive in vitro than Tregs from C57BL/6 mice fed normal chow. Others have suggested that ox-LDL exposure can reduce the suppressive properties of $\mathrm{CD} 4^{+} \mathrm{CD} 25^{+} \mathrm{FoxP}^{+} \mathrm{T}$ cells (81). T cells from hyperlipidemic mice expressed significantly lower levels of the proapoptotic molecule Bim, which has previously been implicated in Treg function, compared with normal mice (82). Changes in Tregs in hyperlipidemic mice also affected tolerance induction based on approaches that require Tregs, such as costimulatory molecule blockade. Indeed, treatment with cytotoxic T lymphocyte antigen-4-Ig (CTLA4-Ig) and anti-CD154 failed to induce tolerance to fully allogeneic cardiac transplants in hyperlipidemic mice (52). Overall, HFDs and consequent hyperlipidemia can impact alloimmune cells directly, or indirectly via increased inflammation (Figure 2), which globally accelerates the kinetics of transplant rejection in nonimmunosuppressed mice and prevents the induction of transplantation tolerance. The impact of hyperlipidemia on tolerance induction or immunosuppression withdrawal in the clinic remains to be investigated.

\section{Impact of pollutants on graft outcome}

Pollutants may affect the alloimmune response via activation of AHR, a cytoplasmic receptor that serves as an environmental sensor. AHR can be activated by many substances, such as polycyclic aromatic hydrocarbons or products of the microbiota (83-85), leading to the activation of a number of transcription factors (86). Depending on the ligand and duration and context of exposure, AHR activation may lead to opposing immune functions $(84,87)$. In an alloimmunity model, exposure to the AHR ligand 2,3,7,8-tetrachlorodibenzo-p-dioxin (TCDD) prolonged skin allograft survival in a fully allogeneic BALB/c into C57BL/6 transplant model, while exposure to the AHR ligand formylindolo (3,2-b)carbazole (FICZ) accelerated rejection (88). These outcomes were associated with expansion of Tregs and increases in IL-17-producing cells, respectively. TCDD administration also prolonged graft survival in a fully allogeneic MHC-mismatched cardiac transplant model (89). In a murine islet cell transplant model, oral administration of VAG539, a pro-agent that is converted to the AHR ligand VAF347, led to long-term graft acceptance in $69 \%$ of recipients (90). Furthermore, VAG539-induced tolerance was transferable by the adoptive transfer of $\mathrm{CD} 11 \mathrm{c}^{+}$cells isolated from treated recipients. Tolerance induction was associated with expansion of $\mathrm{CD} 4{ }^{+} \mathrm{C}$ D $25^{+}$Foxp $^{+}$Tregs. While TCDD has been shown to inhibit B cell differentiation and antibody production in vitro (91-94), no studies have yet demonstrated a potential benefit in transplantation. In humans, there is some observational evidence that traffic air pollution may shorten the life of lung transplants through the development of bronchiolitis obliterans syndrome (95). Overall, AHR may play an important role in transplant homeostasis, and further understanding of its fine balance is required for the development of novel potential therapeutic targets.

Smoking is a major source of toxic substances and has been associated with greater risk of allograft loss (96-98). Smoking may induce vascular damage in the graft $(99,100)$ but may also increase the rate of rejection $(98,101)$. In a murine model of islet 
cell transplantation, second-hand smoking prevented tolerance induction by CD154 costimulation blockade through suppression of indoleamine 2,3-dioxygenase (IDO) (102). Although Treg numbers were not directly affected, overexpression of IDO in islets restored long-term allograft survival. Thus, smoking limits graft survival likely through multiple mechanisms.

Carbon monoxide (CO) can be toxic at high levels because of its 200 times greater affinity for hemoglobin compared with oxygen. However, $\mathrm{CO}$ at low concentrations was found to have cytoprotective effects through binding of iron-containing enzymes such as cytochrome $\mathrm{P} 450$. Using CO-containing preservation solution, Nakao et al. demonstrated reduced inflammation and increased graft survival in treated kidneys prior to transplant surgery (103). Similar protection was observed with incubation of lung and intestinal transplants with low concentrations of $\mathrm{CO}$ (5\%) and was associated with reduced intragraft inflammation following ischemia/reperfusion injury (IRI) $(104,105)$. Despite these experimental data, no clinical trial has yet proven the benefit of $\mathrm{CO}$ administration to explanted grafts prior to transplantation, as an initial registered trial on ClinicalTrials.gov in 2007 was not completed (NCT00531856).

\section{Impact of microbes on alloimmunity and transplant outcome}

Another ubiquitous environmental factor that influences immune responses is the microbiota. Different microbial communities colonize each body site, dependent on oxygen content, moisture, oil composition, etc. (106). The particular microbial species in any given community are greatly influenced by environmental factors such as diet, atmospheric content, and drugs administered. Each microbial community may influence local and/or distal immunity differently, as well as metabolize dietary components or drugs differently. Maintenance of a diverse microbiota in adults is usually correlated with health, which itself may require the right balance of microbes that promote regulatory immune responses (as has been shown for consortia of Clostridium species [refs. 107, 108] and Bacteroides fragilis [ref. 109]) versus conventional effector functions (as has been shown for the mouse commensal segmented filamentous bacterium; refs. 110, 111).

States of altered microbial composition, termed dysbiosis, have been associated with pathological conditions such as obesity and inflammatory bowel disease and shown in some cases to be sufficient to cause pathology (112-114), prompting interest in determining whether microbial communities could impact transplant outcomes and whether they could be manipulated to influence graft stability. One hint that the microbiota may affect graft outcome is the clinical and experimental observation that microbial-colonized organs such as the intestine or the lung display worse outcomes following transplantation than sterile organs such as the heart and the kidney (115), although other features common to lung and intestine, including their higher load in donor lymphoid cells compared with other organs, may play a role in the shorter half-lives of these grafts.

Analysis of the microbiota has mostly focused on bacterial communities, using either cultivation techniques, amplicon sequencing of hypervariable regions in the $16 \mathrm{~S}$ rRNA gene, or shotgun metagenome sequencing of the microbial genome (116). In thinking about the microbiota in transplant hosts, one has to keep in mind that the pathology underlying the need for organ replacement may lead to dysbiosis prior to transplantation. For instance, using cultivation of select intestinal species, the severity of liver disease in liver transplant candidates was inversely correlated with the ratio of Bifidobacterium/Enterococcus species (117). Using quantitative metagenomics, liver cirrhosis was associated with an increase in gut bacterial species of buccal origin, and a discrete set of bacterial genes and their predicted functions appeared distinct from other pathologies, and were diagnostic of cirrhosis (118). Moreover, the microbiota of the donor organ, and not only of the host, may theoretically impact the immune response to the graft. Initial microbiome studies in the transplant field have focused on describing changes in microbial communities after transplantation and observing whether there are any signatures associated with rejection episodes.

Influence of transplantation on the microbiota. Many factors could theoretically have an effect on microbial composition after transplantation, including administration of prophylactic antibiotics or antivirals, administration of immunosuppressive drugs, the initial graft damage from IRI, and the alloimmune response to the transplanted organ. Regardless of the cause, shifts in bacterial communities have been repeatedly reported in fecal, oral, or urine samples of transplant hosts (119-124), most often involving loss of microbial diversity.

Few studies have directly addressed the impact of immunosuppression on the microbiota. Administration of one dose of the lymphocyte-depleting agent alemtuzumab in healthy cynomolgus monkeys perturbed the microbial composition of the intestine, with increased bacterial and fungal richness correlating with decreased numbers of intestinal $\mathrm{T}$ cells, and a return to pretreatment flora as $\mathrm{T}$ cells repopulated the animals $(125,126)$. In mice, administration of prednisolone reduced the Bacteroidetes/Firmicutes ratio, diminished the intestinal antimicrobial defensins RegIII $\gamma$ and IL-22, and induced overgrowth of commensal $E$. coli and colonization by uropathogenic E. coli (127). In contrast, changes following administration of mycophenolate mofetil or the calcineurin inhibitor tacrolimus were more subtle and individual-dependent. Studies in rats and liver transplant patients have associated administration of tacrolimus with increased intestinal permeability in the short term $(128,129)$, which may correct itself at $2-3$ years after transplantation (130). Gingival overgrowth is a common complication of treatment with the other calcineurin inhibitor cyclosporin A and may be due to salivary microbial overgrowth (131), perhaps because of reduced immune-imposed colonization restrictions. Long-term immunosuppression in transplant patients was associated with changes in the salivary bacteriome, increased bacterial richness, and outgrowth of potentially opportunistic pathogens (132). Systemic immunosuppression also increased the total load of the virome, the analysis of which might be useful to monitor immunocompetence (133). Conversely, the microbiota may affect immunosuppression dose requirements. A recent study identified significantly greater abundance of fecal Faecalibacterium prausnitzii in kidney transplant recipients requiring tacrolimus dose escalation than in patients with stable dosing (134). The mechanism by which F prausnitzii may impact, or if it is a biomarker of other factors that impact, tacrolimus metabolism or intestinal absorption remains to be investigated. 
Microbiota and transplant rejection. Changes in intestinal bacterial composition have been associated with acute rejection in animal models and patients, though whether this is a cause or a consequence of rejection is not known. Following liver transplantation, rats develop an increase in the Bacteroidetes/ Firmicutes ratio prior to acute rejection, which correlates with increased intestinal permeability and endotoxin levels (135, 136), suggesting that microbial variation may be used as an early predictor of graft rejection. Interestingly, ischemia preconditioning prior to transplantation, a technique shown to improve graft function, also improved intestinal barrier function and restored intestinal microbiota (137). In human intestinal transplantation, a reduction in Firmicutes was associated with acute rejection (138). One study in kidney transplant patients identified a clear separation in the gut microbial composition of 3 patients who developed acute rejection versus 23 who did not (139), although other studies have argued that interpersonal variability may be too great in many cases to find recurrent characteristics of rare clinical events, and therefore longitudinal patient follow-up may be more indicative of arising problems (124) than interpersonal comparisons. Longitudinally in lung transplantation, a return to pretransplant microbial composition, especially a return of Pseudomonas in patients with cystic fibrosis, was associated with protection against bronchiolitis obliterans syndrome (140), though some species of Pseudomonas may be protective while others may be detrimental to bronchiolitis obliterans syndrome (141).

Using a murine skin transplantation model, Lei et al. showed that pretreatment with broad-spectrum antibiotics (Abx) or use of germ-free mice resulted in prolonged allograft survival (142). Mechanistically, Abx pretreatment was associated with a diminished APC capacity to activate alloreactive T cells, resulting in reduced intragraft $\mathrm{T}$ cell infiltration. Intriguingly, microbial communities from control but not from Abx-pretreated mice restored the priming potential of APCs from germ-free mice (142), indicating vastly distinct alloimmune-modulating effects by different microbial communities. In liver transplantation, the microbiota was shown to upregulate immune responses by increasing Kupffer cell numbers, functional activity, and maturation status, which were decreased in germ-free and Abx-treated mice (143), perhaps by enabling portal circulation of microbial molecular patterns from the gut to the liver.

Microbial products and transplant outcome. The mechanisms by which some microbial communities may influence alloimmunity remain to be determined. Several studies have linked increased intestinal permeability with worse transplant outcomes, suggesting that microbial products that circulate after transplantation may modulate local and perhaps distal alloimmunity. Microbial products may include molecular patterns recognized by pattern-recognition receptors (PRRs) expressed on hematopoietic and nonhematopoietic cells, or metabolic products that may affect the immune system by a variety of mechanisms. Indeed, absence in both donor and recipient mice of MyD88, an adaptor molecule downstream of most TLRs, a family of PRRs, resulted in prolonged survival of minor antigen-mismatched skin grafts (144), although whether this was due to reduced sensing of damage- or of microbe-associated molecular patterns remains to be resolved.
Metabolic products are often codependent on dietary nutrients, microbial composition, and host enzymatic activities (145). In particular, the functional output of the gut microbiota, including short-chain fatty acids (SCFAs), amino acids, and secondary biliary acids, is thought to modulate the pathogenesis of obesity (146). The known roles of some metabolites in immune cell function support their possible involvement in modulating alloimmunity. For instance, bile acids were recently reported to reduce inflammation and metabolic syndrome by inhibiting the NLRP3 inflammasome (147). SCFAs induced the differentiation of colonic Tregs and maintained gut Treg homeostasis (148, 149). A reduction in SCFA butyrate was observed after bone marrow transplantation (BMT), and its administration, or that of butyrate-producing Clostridia, improved intestinal epithelial cell junctional integrity and reduced GVHD (150). Moreover, SCFA administration reduced kidney injury in a mouse model of IRI, an outcome associated with decreased DC maturation and T cell proliferation (151). Whether these properties of select bacterial metabolites can be co-opted to improve graft outcomes should be investigated.

Modifying the microbiota to improve transplant outcomes. Several approaches have been or can be undertaken to modify the microbiota with potential impact on transplant outcome, though the mechanisms linking the changes in microbial species to alloimmunity and graft fate are difficult to tease out. Antibiotics are likely the most effective way to massively change microbial composition. In pediatric BMT, total gastrointestinal decontamination of allograft recipients has been used to prevent acute GVHD. However, the benefit of this approach has been challenged with the demonstration that loss of microbiota diversity is associated with increased lethality in adult BMT (152). Although Abx pretreatment reduced alloimmunity and promoted solid graft survival in mice (142), a more precise understanding of the specific bacteria that promote rejection, and perhaps identification of bacteria that antagonize rejection and that one may want to retain or repopulate after transplantation, are needed. Also, because some bacteria depend on one another for survival (153) or antagonize one another (154), even narrow-spectrum Abx may have unintended consequences that will need to be studied.

Administration of prebiotics (nutrients that sustain growth of select species) and of probiotics (select bacterial species) is another approach to modify the microbiota. Although administration of probiotics may seem like adding "a drop in the bucket," feeding of Bifidobacterium species in mice that lacked this genus was recently shown to allow establishment of these species in the gut and improve antitumor immunity in a mouse model of melanoma (155). In transplantation, administration of probiotics reduced liver damage in rats following IRI (156) or liver transplantation and correlated with increased intestinal Tregs (157). Similarly, a meta-analysis of four clinical trials pointed to the benefit of administering probiotics before or on the day of liver transplantation in patients (158), with a combination of prebiotics and probiotics being possibly superior to probiotics alone, and superior to antibiotic-induced intestinal decontamination (159-161). In malnourished rats, even late probiotic administration several weeks after transplantation was effective at improving intestinal barrier function (162). 
Modifying the diet is another approach to altering the microbiota, though precise control will be difficult to achieve. Nevertheless, in mice with a genetic alteration that resulted in arthritis and spontaneous development of osteomyelitis that correlated with intestinal outgrowth of Prevotella, a diet rich in fat and cholesterol decreased levels of gut Prevotella, reduced systemic pro-IL-1 $\beta$, and prevented bone erosion (163). This demonstrates that diet can have a dominant effect over genetic predisposition.

Impact of pathogenic infections on alloimmunity. Although infections are a less ubiquitous environmental factor than commensals, their occurrence may also impact graft outcome. Indeed, infections prior to transplantation can generate memory $\mathrm{T}$ cells that in some cases cross-react with alloantigens, a phenomenon termed heterologous immunity $(164,165)$. This increases the frequency of alloreactive $\mathrm{T}$ cells and reduces their susceptibility to immunosuppression. At the time of transplantation, viral infections, bacterial infections, and microbial products have all been shown to prevent the induction of tolerance by costimulation blockade therapies (reviewed in ref. 166). Even after establishment of stable tolerance, infection with Listeria monocytogenes was shown to precipitate rejection in a subset of mice by eliciting inflammatory cytokines that could increase alloreactivity (167). Supporting the clinical relevance of these observations, tolerant patients who went on to reject their graft after years of stability experienced viral or bacterial infections prior to the rejection event (168).

\section{Conclusions}

In conclusion, HSD, HFD, and associated hyperlipidemia and select communities of commensal microbes can all promote graft rejection, whereas different pollutants can be either immuno- potentiating or immunosuppressive. The impact of environmental factors on alloimmunity and graft outcome is just beginning to emerge, but initial studies addressing how the environment can affect graft fate are providing new insights into pathways that fine-tune $\mathrm{T}$ cell responses and alloimmunity, and into possible therapeutic interventions that may improve graft survival. However, many gaps remain in the understanding of the cellular and molecular mechanisms underlying the observed effects. Because the microbiota is exquisitely sensitive to changes in diet and other environmental factors, whether changes in microbial communities may be in part responsible for the effect of other environmental factors (Figure 2) remains to be studied. Additionally, most studies to date have addressed the impact of environmental factors on the initial priming of naive alloreactive $\mathrm{T}$ cells. Whether and how these signals affect alloimmunity in sensitized hosts or during ongoing immune responses or established tolerance is an open area of investigation. Finally, how the immune system integrates cues from donor/host genetic disparities in conjunction with the mounting number of environmental factors that can modulate alloimmunity will take many years to unravel.

\section{Acknowledgments}

This work was supported in part by American Heart Association grant 12FTF120070328 to LVR, NIH grant R01-AI116714 and Grant-in-Aid 7-04-RA-45 from the American Heart Association to JI, and R01-AI115716 to MLA.

Address correspondence to: Maria-Luisa Alegre, Department of Medicine, The University of Chicago, 924 E. 57th Street, JFK-R312, Chicago, Illinois 60637, USA. Phone: 773.834.4317; Email:malegre@midway.uchicago.edu.
1. Kotchen TA, Cowley AW Jr, Frohlich ED. Salt in health and disease - a delicate balance. $N$ Engl J Med. 2013;368(13):1229-1237.

2. Go WY, Liu X, Roti MA, Liu F, Ho SN. NFAT5/ TonEBP mutant mice define osmotic stress as a critical feature of the lymphoid microenvironment. Proc Natl Acad SciU S A. 2004;101(29):10673-10678.

3. Kopp C, et al. 23Na magnetic resonance imaging-determined tissue sodium in healthy subjects and hypertensive patients. Hypertension. 2013;61(3):635-640.

4. Machnik A, et al. Macrophages regulate saltdependent volume and blood pressure by a vascular endothelial growth factor-C-dependent buffering mechanism. Nat Med. 2009;15(5):545-552.

5 . Wiig $\mathrm{H}$, et al. Immune cells control skin lymphatic electrolyte homeostasis and blood pressure. J Clin Invest. 2013;123(7):2803-2815.

6. Jantsch J, et al. Cutaneous $\mathrm{Na}^{+}$storage strengthens the antimicrobial barrier function of the skin and boosts macrophage-driven host defense. Cell Metab. 2015;21(3):493-501.

7. Binger KJ, et al. High salt reduces the activation of IL-4- and IL-13-stimulated macrophages. J Clin Invest. 2015;125(11):4223-4238.

8. Kleinewietfeld M, et al. Sodium chloride drives autoimmune disease by the induction of pathogenic TH17 cells. Nature. 2013;496(7446):518-522.
9. Wu C, et al. Induction of pathogenic TH17 cells by inducible salt-sensing kinase SGK1. Nature. 2013;496(7446):513-517.

10. Berer K, et al. Commensal microbiota and myelin autoantigen cooperate to trigger autoimmune demyelination. Nature. 2011;479(7374):538-541.

11. Farez MF, Fiol MP, Gaitán MI, Quintana FJ, Correale J. Sodium intake is associated with increased disease activity in multiple sclerosis. J Neurol Neurosurg Psychiatr. 2015;86(1):26-31.

12. Safa K, et al. Salt accelerates allograft rejection through serum- and glucocorticoid-regulated kinase-1-dependent inhibition of regulatory $\mathrm{T}$ cells. J Am Soc Nephrol. 2015;26(10):2341-2347.

13. Hernandez AL, et al. Sodium chloride inhibits the suppressive function of $\mathrm{FOXP}^{+}$regulatory T cells. J Clin Invest. 2015;125(11):4212-4222.

14. Stehlik J, et al. The Registry of the International Society for Heart and Lung Transplantation: Twenty-eighth Adult Heart Transplant Report - 2011. J Heart Lung Transplant. 2011;30(10):1078-1094.

15. Tuttolomondo A, Di Raimondo D, Pecoraro R, Arnao V, Pinto A, Licata G. Atherosclerosis as an inflammatory disease. Curr Pharm Des. 2012;18(28):4266-4288.

16. Libby P, Ridker PM, Hansson GK. Progress and challenges in translating the biology of atherosclerosis. Nature. 2011;473(7347):317-325.
17. Ng HP, Burris RL, Nagarajan S. Attenuated atherosclerotic lesions in apoE-Fc $\gamma$-chain-deficient hyperlipidemic mouse model is associated with inhibition of Th17 cells and promotion of regulatory T cells. J Immunol. 2011;187(11):6082-6093.

18. Gao Q, et al. A critical function of Th17 proinflammatory cells in the development of atherosclerotic plaque in mice. J Immunol. 2010;185(10):5820-5827.

19. Bilchick KC, Henrikson CA, Skojec D, Kasper EK, Blumenthal RS. Treatment of hyperlipidemia in cardiac transplant recipients. Am Heart J. 2004;148(2):200-210.

20. Stone NJ, et al. 2013 ACC/AHA guideline on the treatment of blood cholesterol to reduce atherosclerotic cardiovascular risk in adults: a report of the American College of Cardiology/American Heart Association Task Force on Practice Guidelines. Circulation. 2014;129(25 suppl 2):S1-S45.

21. Pflugfelder PW, Huff M, Oskalns R, Rudas L, Kostuk WJ. Cholesterol-lowering therapy after heart transplantation: a 12-month randomized trial. J Heart Lung Transplant. 1995;14(4):613-622.

22. Zakliczynski M, et al. In the era of the universal use of statins dyslipidemia's are still common in heart transplant recipients: a cross-sectional study. Transplant Proc. 2011;43(8):3071-3073.

23. Sánchez Lázaro IJ, et al. Influence of traditional cardiovascular risk factors in the recipient on the 
development of cardiac allograft vasculopathy after heart transplantation. Transplant Proc. 2008;40(9):3056-3057.

24. Bae JH, et al. Association of angiotensin-converting enzyme inhibitors and serum lipids with plaque regression in cardiac allograft vasculopathy. Transplantation. 2006;82(8):1108-1111.

25. Radovancevic B, et al. Factors predicting 10-year survival after heart transplantation. J Heart Lung Transplant. 2005;24(2):156-159.

26. Khatri P, et al. A common rejection module (CRM) for acute rejection across multiple organs identifies novel therapeutics for organ transplantation. JExp Med. 2013;210(11):2205-2221.

27. Holdaas $\mathrm{H}$, et al. Effect of fluvastatin on cardiac outcomes in renal transplant recipients: a multicentre, randomised, placebo-controlled trial. Lancet. 2003;361(9374):2024-2031.

28. Shimizu K, Aikawa M, Takayama K, Libby P, Mitchell RN. Direct anti-inflammatory mechanisms contribute to attenuation of experimental allograft arteriosclerosis by statins. Circulation . 2003;108(17):2113-2120.

29. Yi T, et al. Amelioration of human allograft arterial injury by atorvastatin or simvastatin correlates with reduction of interferon-gamma production by infiltrating $\mathrm{T}$ cells. Transplantation . 2008;86(5):719-727.

30. Zhang J, et al. Statins directly suppress cytokine production in murine intraepithelial lymphocytes. Cytokine. 2013;61(2):540-545.

31. Kwak B, Mulhaupt F, Myit S, Mach F. Statins as a newly recognized type of immunomodulator. Nat Med. 2000;6(12):1399-1402.

32. Yilmaz A, et al. Differential effects of statins on relevant functions of human monocyte-derived dendritic cells. J Leukoc Biol. 2006;79(3):529-538.

33. Youssef S, et al. The HMG-CoA reductase inhibitor, atorvastatin, promotes a Th2 bias and reverses paralysis in central nervous system autoimmune disease. Nature. 2002;420(6911):78-84.

34. Aktas $\mathrm{O}$, et al. Treatment of relapsing paralysis in experimental encephalomyelitis by targeting Th1 cells through atorvastatin. JExp Med. 2003;197(6):725-733.

35. Wang $\mathrm{Y}$, et al. Blocking LFA-1 activation with lovastatin prevents graft-versus-host disease in mouse bone marrow transplantation. Biol Blood Marrow Transplant. 2009;15(12):1513-1522.

36. Greenwood J, et al. Lovastatin inhibits brain endothelial cell Rho-mediated lymphocyte migration and attenuates experimental autoimmune encephalomyelitis. FASEB $J$. 2003;17(8):905-907.

37. Ghittoni R, Lazzerini PE, Pasini FL, Baldari CT. T lymphocytes as targets of statins: molecular mechanisms and therapeutic perspectives. Inflamm Allergy Drug Targets. 2007;6(1):3-16.

38. Li XL, et al. Atorvastatin ameliorates experimental autoimmune neuritis by decreased Th1/Th17 cytokines and up-regulated $\mathrm{T}$ regulatory cells. Cell Immunol. 2011;271(2):455-461.

39. Zhang X, Jin J, Peng X, Ramgolam VS, MarkovicPlese S. Simvastatin inhibits IL-17 secretion by targeting multiple IL-17-regulatory cytokines and by inhibiting the expression of IL-17 transcription factor RORC in CD4 $4^{+}$lymphocytes. JImmunol. 2008;180(10):6988-6996.
40. Ghittoni R, et al. Simvastatin inhibits T-cell activation by selectively impairing the function of Ras superfamily GTPases. FASEB J. 2005;19(6):605-607.

41. Dunn SE, et al. Isoprenoids determine Th1/Th2 fate in pathogenic $\mathrm{T}$ cells, providing a mechanism of modulation of autoimmunity by atorvastatin. JExp Med. 2006;203(2):401-412.

42. Piedrahita JA, Zhang SH, Hagaman JR, Oliver PM, Maeda N. Generation of mice carrying a mutant apolipoprotein $\mathrm{E}$ gene inactivated by gene targeting in embryonic stem cells. Proc Natl Acad Sci U S A. 1992;89(10):4471-4475.

43. Whitman SC. A practical approach to using mice in atherosclerosis research. Clin Biochem Rev. 2004;25(1):81-93.

44. Plump AS, Breslow JL. Apolipoprotein E and the apolipoprotein E-deficient mouse. Annu Rev Nutr. 1995;15:495-518.

45. Yuan J, Bagley J, Iacomini J. Hyperlipidemia promotes anti-donor Th17 responses that accelerate allograft rejection. Am J Transplant. 2015;15(9):2336-2345.

46. Moghadasian MH, et al. Pathophysiology of apolipoprotein $\mathrm{E}$ deficiency in mice: relevance to apo E-related disorders in humans. FASEB J. 2001;15(14):2623-2630.

47. Davis HR, et al. Deficiency of Niemann-Pick C1 Like 1 prevents atherosclerosis in $\mathrm{ApoE}^{-/ /}$mice. Arterioscler Thromb Vasc Biol. 2007;27(4):841-849.

48. Smith $\mathrm{E}$, et al. Blockade of interleukin-17A results in reduced atherosclerosis in apolipoprotein E-deficient mice. Circulation. 2010;121(15):1746-1755.

49. Ali K, Middleton M, Puré E, Rader DJ. Apolipoprotein $\mathrm{E}$ suppresses the type I inflammatory response in vivo. Circ Res. 2005;97(9):922-927.

50. de Bont N, Netea MG, Demacker PN, Kullberg BJ, van der Meer JW, Stalenhoef AF. Apolipoprotein E-deficient mice have an impaired immune response to Klebsiella pneumoniae. Eur JClin Invest. 2000;30(9):818-822.

51. Van Oosten M, et al. Apolipoprotein E protects against bacterial lipopolysaccharide-induced lethality. A new therapeutic approach to treat gram-negative sepsis. J Biol Chem. 2001;276(12):8820-8824.

52. Bagley J, Yuan J, Chandrakar A, Iacomini J. Hyperlipidemia alters regulatory $\mathrm{T}$ cell function and promotes resistance to tolerance induction through costimulatory molecule blockade. $\mathrm{Am} J$ Transplant. 2015;15(9):2324-2335.

53. Molinero LL, et al. High-fat diet-induced obesity enhances allograft rejection. Transplantation. 2016;100(5):1015-1021.

54. Dimeloe S, Burgener AV, Grählert J, Hess C. $\mathrm{T}$-cell metabolism governing activation, proliferation and differentiation; a modular view. Immunology. 2017;150(1):35-44.

55. Armstrong AJ, Gebre AK, Parks JS, Hedrick CC. ATP-binding cassette transporter G1 negatively regulates thymocyte and peripheral lymphocyte proliferation. JImmunol. 2010;184(1):173-183.

56. Kidani Y, et al. Sterol regulatory element-binding proteins are essential for the metabolic programming of effector $\mathrm{T}$ cells and adaptive immunity. Nat Immunol. 2013;14(5):489-499.

57. Yang $\mathrm{W}$, et al. Potentiating the antitumour response of CD8(+) T cells by modulating cholesterol metab- olism. Nature. 2016;531(7596):651-655.

58. Martínez-Martín N, et al. Cooperativity between $\mathrm{T}$ cell receptor complexes revealed by conformational mutants of CD3e. Sci Signal. 2009;2(83):ra43.

59. Wang F, Beck-García K, Zorzin C, Schamel WW, Davis MM. Inhibition of $\mathrm{T}$ cell receptor signaling by cholesterol sulfate, a naturally occurring derivative of membrane cholesterol. Nat Immunol. 2016;17(7):844-850.

60. Cui G, et al. Liver X receptor (LXR) mediates negative regulation of mouse and human Th17 differentiation. JClin Invest. 2011;121(2):658-670.

61. Soroosh P, et al. Oxysterols are agonist ligands of ROR $\gamma$ t and drive Th17 cell differentiation. Proc Natl Acad Sci U S A. 2014;111(33):12163-12168.

62. Zeng H, Yang K, Cloer C, Neale G, Vogel P, Chi H. mTORC1 couples immune signals and metabolic programming to establish $\mathrm{T}(\mathrm{reg})$-cell function. Nature. 2013;499(7459):485-490.

63. Cheng HY, et al. Loss of ABCG1 influences regulatory $\mathrm{T}$ cell differentiation and atherosclerosis. JClin Invest. 2016;126(9):3236-3246.

64. Maganto-García E, Tarrio ML, Grabie N, Bu DX, Lichtman AH. Dynamic changes in regulatory $\mathrm{T}$ cells are linked to levels of diet-induced hypercholesterolemia. Circulation. 2011;124(2):185-195.

65. Ghosh J, et al. Hyperlipidemia offers protection against Leishmania donovani infection role of membrane cholesterol. J Lipid Res. 2012;53(12):2560-2572.

66. Wang SH, Yuan SG, Peng DQ, Zhao SP. High-density lipoprotein affects antigen presentation by interfering with lipid raft: a promising anti-atherogenic strategy. Clin Exp Immunol. 2010;160(2):137-142.

67. Sag D, Cekic C, Wu R, Linden J, Hedrick CC. The cholesterol transporter ABCG1 links cholesterol homeostasis and tumour immunity. Nat Commun. 2015;6:6354.

68. Wojcik AJ, Skaflen MD, Srinivasan S, Hedrick CC. A critical role for ABCG1 in macrophage inflammation and lung homeostasis. J Immunol. 2008;180(6):4273-4282.

69. Yvan-Charvet L, et al. Increased inflammatory gene expression in $\mathrm{ABC}$ transporter-deficient macrophages: free cholesterol accumulation, increased signaling via toll-like receptors, and neutrophil infiltration of atherosclerotic lesions. Circulation. 2008;118(18):1837-1847.

70. Choi SH, et al. SYK regulates macrophage MHC-II expression via activation of autophagy in response to oxidized LDL. Autophagy. 2015;11(5):785-795.

71. Perrin-Cocon L, Coutant F, Agaugué S, Deforges S, André P, Lotteau V. Oxidized low-density lipoprotein promotes mature dendritic cell transition from differentiating monocyte. J Immunol. 2001;167(7):3785-3791.

72. Yu K, et al. Disruption of the TSLP-TSLPR-LAP signaling between epithelial and dendritic cells through hyperlipidemia contributes to regulatory $\mathrm{T}$-Cell defects in atherosclerotic mice. Atherosclerosis. 2015;238(2):278-288.

73. Packard RR, Maganto-García E, Gotsman I, Tabas I, Libby P, Lichtman AH. CD11c(+) dendritic cells maintain antigen processing, presentation capabilities, and CD4(+) T-cell 
priming efficacy under hypercholesterolemic conditions associated with atherosclerosis. Circ Res. 2008;103(9):965-973.

74. Lim HY, et al. Hypercholesterolemic mice exhibit lymphatic vessel dysfunction and degeneration. Am J Pathol. 2009;175(3):1328-1337.

75. Tenger C, Zhou X. Apolipoprotein E modulates immune activation by acting on the antigenpresenting cell. Immunology. 2003;109(3):392-397.

76. Baitsch D, et al. Apolipoprotein E induces antiinflammatory phenotype in macrophages. Arterioscler Thromb Vasc Biol. 2011;31(5):1160-1168.

77. Grainger DJ, Reckless J, McKilligin E. Apolipoprotein $\mathrm{E}$ modulates clearance of apoptotic bodies in vitro and in vivo, resulting in a systemic proinflammatory state in apolipoprotein E-deficient mice. J Immunol. 2004;173(10):6366-6375.

78. James BR, Tomanek-Chalkley A, Askeland EJ, Kucaba T, Griffith TS, Norian LA. Diet-induced obesity alters dendritic cell function in the presence and absence of tumor growth. JImmunol. 2012;189(3):1311-1321.

79. Pizzolla A, et al. High fat diet inhibits dendritic cell and $\mathrm{T}$ cell response to allergens but does not impair inhalational respiratory tolerance. PLoS One. 2016;11(8): 0160407.

80. Xie JJ, et al. The Th17/Treg functional imbalance during atherogenesis in ApoE(-/-) mice. Cytokine. 2010;49(2):185-193.

81. Mor A, et al. Role of naturally occurring CD4 $\mathrm{CD} 25^{+}$regulatory $\mathrm{T}$ cells in experimental atherosclerosis. Arterioscler Thromb Vasc Biol. 2007;27(4):893-900.

82. Chougnet CA, et al. A major role for Bim in regulatory $\mathrm{T}$ cell homeostasis. J Immunol. 2011;186(1):156-163.

83. Kiss EA, et al. Natural aryl hydrocarbon receptor ligands control organogenesis of intestinal lymphoid follicles. Science. 2011;334(6062):1561-1565.

84. Veldhoen M, et al. The aryl hydrocarbon receptor links TH17-cell-mediated autoimmunity to environmental toxins. Nature. 2008;453(7191):106-109.

85. Lee JS, et al. AHR drives the development of gut ILC22 cells and postnatal lymphoid tissues via pathways dependent on and independent of Notch. Nat Immunol. 2011;13(2):144-151.

86. Nguyen LP, Bradfield CA. The search for endogenous activators of the aryl hydrocarbon receptor. Chem Res Toxicol. 2008;21(1):102-116.

87. Quintana FJ, et al. Control of T(reg) and $\mathrm{T}(\mathrm{H}) 17$ cell differentiation by the aryl hydrocarbon receptor. Nature. 2008;453(7191):65-71.

88. Pauly SK, Fechner JH, Zhang X, Torrealba J, Bradfield CA, Mezrich JD. The aryl hydrocarbon receptor influences transplant outcomes in response to environmental signals. Toxicol Environ Chem. 2012;94(6):1175-1187.

89. Cai LJ, Yu DW, Gao Y, Yang C, Zhou HM, Chen $\mathrm{ZH}$. Activation of aryl hydrocarbon receptor prolongs survival of fully mismatched cardiac allografts. J Huazhong Univ Sci Technol Med Sci. 2013;33(2):199-204.

90. Hauben E, et al. Activation of the aryl hydrocarbon receptor promotes allograft-specific tolerance through direct and dendritic cell-mediated effects on regulatory T cells. Blood. 2008;112(4):1214-1222.

91. Fernando TM, Ochs SD, Liu J, Chambers-Turner
RC, Sulentic CE. 2,3,7,8-tetrachlorodibenzo-p-dioxin induces transcriptional activity of the human polymorphic hs1,2 enhancer of the 3'Igh regulatory region. JImmunol. 2012;188(7):3294-3306.

92. Sulentic CE, Holsapple MP, Kaminski NE. Putative link between transcriptional regulation of IgM expression by 2,3,7,8-tetrachlorodibenzop-dioxin and the aryl hydrocarbon receptor/dioxin-responsive enhancer signaling pathway. JPharmacol Exp Ther. 2000;295(2):705-716.

93. Sulentic CE, Kaminski NE. The long winding road toward understanding the molecular mechanisms for B-cell suppression by 2,3,7,8-tetrachlorodibenzo-p-dioxin. Toxicol Sci. 2011;120(suppl 1):S171-S191.

94. Yoo BS, Boverhof DR, Shnaider D, Crawford RB, Zacharewski TR, Kaminski NE. 2,3,7,8-Tetrachlorodibenzo-p-dioxin (TCDD) alters the regulation of Pax 5 in lipopolysaccharide-activated B cells. Toxicol Sci. 2004;77(2):272-279.

95. Nawrot TS, et al. The impact of traffic air pollution on bronchiolitis obliterans syndrome and mortality after lung transplantation. Thorax. 2011;66(9):748-754.

96. Ponticelli C, Villa M, Cesana B, Montagnino G, Tarantino A. Risk factors for late kidney allograft failure. Kidney Int. 2002;62(5):1848-1854.

97. Sung RS, Althoen M, Howell TA, Ojo AO, Merion RM. Excess risk of renal allograft loss associated with cigarette smoking. Transplantation. 2001;71(12):1752-1757.

98. Corbett C, Armstrong MJ, Neuberger J. Tobacco smoking and solid organ transplantation. Transplantation. 2012;94(10):979-987.

99. Cosio FG, et al. Patient survival after renal transplantation: II. The impact of smoking. Clin Transplant. 1999;13(4):336-341.

100.Kasiske BL, Klinger D. Cigarette smoking in renal transplant recipients. JAm Soc Nephrol. 2000;11(4):753-759.

101. Nogueira JM, Haririan A, Jacobs SC, Cooper M, Weir MR. Cigarette smoking, kidney function, and mortality after live donor kidney transplant. Am J Kidney Dis. 2010;55(5):907-915.

102. Wan F, Dai H, Zhang S, Moore Y, Wan N, Dai Z. Cigarette smoke exposure hinders long-term allograft survival by suppressing indoleamine 2, 3-dioxygenase expression. Am J Transplant. 2012;12(3):610-619.

103. Nakao A, et al. Ex vivo carbon monoxide prevents cytochrome $\mathrm{P} 450$ degradation and ischemia/ reperfusion injury of kidney grafts. Kidney Int . 2008;74(8):1009-1016.

104. Kohmoto J, et al. Carbon monoxide-saturated preservation solution protects lung grafts from ischemia-reperfusion injury. J Thorac Cardiovasc Surg. 2008;136(4):1067-1075.

105. Nakao A, et al. Ex vivo application of carbon monoxide in University of Wisconsin solution to prevent intestinal cold ischemia/reperfusion injury. Am J Transplant. 2006;6(10):2243-2255.

106. Grice EA, Segre JA. The skin microbiome. Nat Rev Microbiol. 2011;9(4):244-253.

107.Atarashi K, et al. Treg induction by a rationally selected mixture of Clostridia strains from the human microbiota. Nature. 2013;500(7461):232-236.

108. Atarashi K, et al. Induction of colonic regulatory
T cells by indigenous Clostridium species. $\mathrm{Sci}$ ence. 2011;331(6015):337-341.

109. Round JL, Mazmanian SK. Inducible Foxp $3^{+}$ regulatory T-cell development by a commensal bacterium of the intestinal microbiota. Proc Natl Acad Sci U S A. 2010;107(27):12204-12209.

110. Ivanov II, et al. Induction of intestinal Th17 cells by segmented filamentous bacteria. Cell. 2009;139(3):485-498.

111. Gaboriau-Routhiau V, et al. The key role of segmented filamentous bacteria in the coordinated maturation of gut helper $\mathrm{T}$ cell responses. Immunity. 2009;31(4):677-689.

112. Ridaura VK, et al. Gut microbiota from twins discordant for obesity modulate metabolism in mice. Science. 2013;341(6150):1241214.

113. Tlaskalová-Hogenová $\mathrm{H}$, et al. The role of gut microbiota (commensal bacteria) and the mucosal barrier in the pathogenesis of inflammatory and autoimmune diseases and cancer: contribution of germ-free and gnotobiotic animal models of human diseases. Cell Mol Immunol. 2011;8(2):110-120

114. $\mathrm{Chu} \mathrm{H}$, et al. Gene-microbiota interactions contribute to the pathogenesis of inflammatory bowel disease. Science. 2016;352(6289):1116-1120.

115. Alegre ML, Mannon RB, Mannon PJ. The microbiota, the immune system and the allograft. Am J Transplant. 2014;14(6):1236-1248.

116. Ranjan R, Rani A, Metwally A, McGee HS, Perkins DL. Analysis of the microbiome: advantages of whole genome shotgun versus $16 \mathrm{~S}$ amplicon sequencing. Biochem Biophys Res Commun. 2016;469(4):967-977.

117. Grąt M, et al. The relevance of intestinal dysbiosis in liver transplant candidates. Transpl Infect Dis. 2015;17(2):174-184

118. Qin N, et al. Alterations of the human gut microbiome in liver cirrhosis. Nature. 2014;513(7516):59-64.

119. Charlson ES, et al. Lung-enriched organisms and aberrant bacterial and fungal respiratory microbiota after lung transplant. Am J Respir Crit Care Med. 2012;186(6):536-545.

120. Borewicz K, Pragman AA, Kim HB, Hertz M, Wendt C, Isaacson RE. Longitudinal analysis of the lung microbiome in lung transplantation. FEMS Microbiol Lett. 2013;339(1):57-65.

121. Wu ZW, et al. Changes of gut bacteria and immune parameters in liver transplant recipients. HBPD INT. 2012;11(1):40-50.

122.Sheehy EC, Beighton D, Roberts GJ. The oral microbiota of children undergoing liver transplantation. Oral Microbiol Immunol. 2000;15(3):203-210.

123. Rani A, et al. Urinary microbiome of kidney transplant patients reveals dysbiosis with potential for antibiotic resistance [published online ahead of print September 9, 2016]. Transl Res. https://doi.org/10.1016/j.trsl.2016.08.008.

124. Fricke WF, Maddox C, Song Y, Bromberg JS. Human microbiota characterization in the course of renal transplantation. Am J Transplant. 2014;14(2):416-427.

125. Li QR, Wang CY, Tang C, He Q, Li N, Li JS. Reciprocal interaction between intestinal microbiota and mucosal lymphocyte in cynomolgus monkeys after alemtuzumab treatment. Am J Trans- 
plant. 2013;13(4):899-910.

126.Li Q, Wang C, Tang C, He Q, Li J. Lymphocyte depletion after alemtuzumab induction disrupts intestinal fungal microbiota in cynomolgus monkeys. Transplantation. 2014;98(9):951-959.

127. Tourret J, Willing BP, Dion S, MacPherson J, Denamur E, Finlay BB. Immunosuppressive Treatment Alters Secretion of Ileal Antimicrobial Peptides and Gut Microbiota, and Favors Subsequent Colonization by Uropathogenic Escherichia coli. Transplantation. 2017;101(1):74-82.

128. Madsen KL, Yanchar NL, Sigalet DL, Reigel T, Fedorak RN. FK506 increases permeability in rat intestine by inhibiting mitochondrial function. Gastroenterology.1995;109(1):107-114.

129. Gabe SM, et al. The effect of tacrolimus (FK506) on intestinal barrier function and cellular energy production in humans. Gastroenterology. 1998;115(1):67-74.

130. Parrilli G, et al. Effect of chronic administration of tacrolimus and cyclosporine on human gastrointestinal permeability. Liver Transpl. 2003;9(5):484-488.

131. Romito GA, Pustiglioni FE, Saraiva L, Pustiglioni AN, Lotufo RF, Stolf NA. Relationship of subgingival and salivary microbiota to gingival overgrowth in heart transplant patients following cyclosporin A therapy. J Periodontol. 2004;75(7):918-924.

132. Diaz PI, et al. Transplantation-associated long-term immunosuppression promotes oral colonization by potentially opportunistic pathogens without impacting other members of the salivary bacteriome. Clin Vaccine Immunol. 2013;20(6):920-930.

133. De Vlaminck I, et al. Temporal response of the human virome to immunosuppression and antiviral therapy. Cell. 2013;155(5):1178-1187.

134. Lee JR, et al. Gut microbiota and tacrolimus dosing in kidney transplantation. PLOS ONE. 2015;10(3):e0122399.

135. Xie Y, et al. Structural shifts of fecal microbial communities in rats with acute rejection after liver transplantation. Microb Ecol. 2012;64(2):546-554.

136. Ren Z, et al. Intestinal microbial variation may predict early acute rejection after liver transplantation in rats. Transplantation. 2014;98(8):844-852.

137. Ren Z, et al. Liver ischemic preconditioning (IPC) improves intestinal microbiota following liver transplantation in rats through $16 \mathrm{~s}$ rDNA-based analysis of microbial structure shift. PLOS ONE. 2013;8(10):e75950.

138. Oh PL, Martínez I, Sun Y, Walter J, Peterson DA, Mercer DF. Characterization of the ileal microbiota in rejecting and nonrejecting recipients of small bowel transplants. Am J Transplant. 2012;12(3):753-762.

139. Lee JR, et al. Gut microbial community structure and complications after kidney transplantation: a pilot study. Transplantation. 2014;98(7):697-705.

140. Willner DL, et al. Reestablishment of recipient-associated microbiota in the lung allograft is linked to reduced risk of bronchiolitis obliterans syndrome. Am J Respir Crit Care Med. 2013;187(6):640-647.

141. Dickson RP, et al. Changes in the lung microbiome following lung transplantation include the emergence of two distinct Pseudomonas species with distinct clinical associations. PLOS ONE. 2014;9(5):e97214.

142.Lei YM, et al. The composition of the microbiota modulates allograft rejection. JClin Invest. 2016;126(7):2736-2744.

143. Corbitt N, et al. Gut bacteria drive Kupffer cell expansion via MAMP-mediated ICAM-1 induction on sinusoidal endothelium and influence preservation-reperfusion injury after orthotopic liver transplantation. Am J Pathol. 2013;182(1):180-191.

144.Goldstein DR, Tesar BM, Akira S, Lakkis FG. Critical role of the Toll-like receptor signal adaptor protein MyD88 in acute allograft rejection. JClin Invest. 2003;111(10):1571-1578.

145. Heinken A, Thiele I. Systems biology of hostmicrobe metabolomics. Wiley Interdiscip Rev Syst Biol Med. 2015;7(4):195-219.

146.Neis EP, Dejong CH, Rensen SS. The role of microbial amino acid metabolism in host metabolism. Nutrients. 2015;7(4):2930-2946.

147. Guo C, et al. Bile acids control inflammation and metabolic disorder through inhibition of NLRP3 inflammasome. Immunity. 2016;45(4):802-816.

148. Furusawa Y, et al. Commensal microbe-derived butyrate induces the differentiation of colonic regulatory T cells. Nature. 2013;504(7480):446-450.

149.Smith PM, et al. The microbial metabolites, short-chain fatty acids, regulate colonic Treg cell homeostasis. Science. 2013;341(6145):569-573.

150. Mathewson ND, et al. Gut microbiome-derived metabolites modulate intestinal epithelial cell damage and mitigate graft-versus-host disease. Nat Immunol. 2016;17(5):505-513.

151. Andrade-Oliveira V, et al. Gut Bacteria Products Prevent AKI Induced by Ischemia-Reperfusion. J Am Soc Nephrol. 2015;26(8):1877-1888.

152. Whangbo J, Ritz J, Bhatt A. Antibiotic-mediated modification of the intestinal microbiome in allogeneic hematopoietic stem cell transplantation. Bone Marrow Transplant. 2017;52(2):183-190.

153. Rey FE, Gonzalez MD, Cheng J, Wu M, Ahern PP, Gordon JI. Metabolic niche of a prominent sulfate-reducing human gut bacterium. Proc Natl Acad Sci U S A. 2013;110(33):13582-13587.

154. Roelofs KG, Coyne MJ, Gentyala RR, Chatzidaki-Livanis M, Comstock LE. Bacteroidales secreted antimicrobial proteins target surface molecules necessary for gut colonization and mediate com- petition in vivo. MBio. 2016;7(4):e01055-16.

155. Sivan A, et al. Commensal Bifidobacterium promotes antitumor immunity and facilitates anti-PD-L1 efficacy. Science. 2015;350(6264):1084-1089.

156. Xing HC, et al. Protective role of supplement with foreign Bifidobacterium and Lactobacillus in experimental hepatic ischemia-reperfusion injury. J Gastroenterol Hepatol. 2006;21(4):647-656.

157. Xie Y, et al. Effect of intestinal microbiota alteration on hepatic damage in rats with acute rejection after liver transplantation. Microb Ecol. 2014;68(4):871-880.

158. Sawas T, Al Halabi S, Hernaez R, Carey WD, Cho WK. Patients receiving prebiotics and probiotics before liver transplantation develop fewer infections than controls: a systematic review and meta-analysis. Clin Gastroenterol Hepatol. 2015;13(9):1567-1574.e3; quiz e143.

159. Rayes N, et al. Early enteral supply of lactobacillus and fiber versus selective bowel decontamination: a controlled trial in liver transplant recipients. Transplantation. 2002;74(1):123-127.

160. Rayes N, et al. Supply of pre- and probiotics reduces bacterial infection rates after liver transplantation - a randomized, double-blind trial. Am J Transplant. 2005;5(1):125-130.

161. Eguchi S, Takatsuki M, Hidaka M, Soyama A, Ichikawa T, Kanematsu T. Perioperative synbiotic treatment to prevent infectious complications in patients after elective living donor liver transplantation: a prospective randomized study. $\mathrm{Am} \mathrm{J}$ Surg. 2011;201(4):498-502.

162.Ren ZG, et al. Protective effect of probiotics on intestinal barrier function in malnourished rats after liver transplantation. HBPD INT 2011;10(5):489-496.

163. Lukens JR, et al. Dietary modulation of the microbiome affects autoinflammatory disease. Nature. 2014;516(7530):246-249.

164. Adams AB, et al. Heterologous immunity provides a potent barrier to transplantation tolerance. J Clin Invest. 2003;111(12):1887-1895.

165. Brehm MA, et al. Allografts stimulate cross-reactive virus-specific memory $\mathrm{CD} 8 \mathrm{~T}$ cells with private specificity. Am J Transplant. 2010;10(8):1738-1748.

166. Chong AS, Alegre ML. The impact of infection and tissue damage in solid-organ transplantation. Nat Rev Immunol. 2012;12(6):459-471.

167. Wang $\mathrm{T}$, et al. Infection with the intracellular bacterium, Listeria monocytogenes, overrides established tolerance in a mouse cardiac allograft model. Am J Transplant. 2010;10(7):1524-1533.

168. Brouard S, et al. The natural history of clinical operational tolerance after kidney transplantation through twenty-seven cases. Am J Transplant. 2012;12(12):3296-3307. 